\title{
Bevacizumab in diabetic macular edema. A retrospective analysis of the efficacy of treatment in a group of 100 patients
}

\author{
Piotr Kanclerz' , Andrzej Grzybowskii ${ }^{2,3}$, Rafał Ściegienny ${ }^{1}$ \\ 'Department of Ophthalmology, Medical University of Gdansk, Gdansk, Poland \\ ${ }^{2}$ Department of Ophthalmology, Poznan City Hospital, Poznan, Poland \\ ${ }^{3}$ Department of Ophthalmology, University of Warmia and Mazury, Olsztyn, Poland
}

\begin{abstract}
INTRODUCTION. The aim of the study was to retrospectively evaluate the efficacy of intravitreal injections of bevacizumab in a large group of diabetic macular edema (DME) patients.

MATERIALS AND METHODS. One hundred patients (125 eyes) suffering from diabetes type 1 or 2 and DME were treated with two or three monthly 1.25 -mg intravitreal injections of bevacizumab.

RESULTS. The mean (SD) best correct visual acuity (BCVA) before treatment was $63.8( \pm 26.2)$ letters. At months 1,2 , and 3 the mean change (SE) in BCVA from baseline was $+4.4( \pm 0.49),+4.9(0.38)$, and $+3.1( \pm 0.39)$ letters, respectively. The mean macular retinal thickness dropped from $457( \pm 183)$ microns to $308( \pm 122)$ microns after three months. The improvement of retinal morphology in optical coherence tomography was evident: a decrease in the number of intraretinal cysts and a generalised shrinkage of retinal macular edema. There were no serious adverse effects of the treatment.

CONCLUSION. The majority of patients with DME showed an improvement in visual acuity, particularly after the first injection of bevacizumab. Bevacizumab therapy is an interesting alternative to DME treatment.

KEY WORDS: bevacizumab, diabetic retinopathy, intravitreal injections, macular edema, optical coherence tomography, vascular endothelial growth factor $\mathrm{A}$
\end{abstract}

Ophthalmol J 2017; Vol. 2, No. 1, 1-5

\section{INTRODUCTION}

Diabetic macular edema (DME) is an accumulation of fluid in the outer plexiform layer and inner nuclear retina as a result of leakage of retinal microvessels. It may occur in any stage of diabetic retinopathy (DR), which is the most common microvascular complication of both types of diabetes. The majority of chronic patients will develop retinopathy with DME. If the edema affects the central part of the macula, the patient loses visual acuity. This is the most common symptom of DME [1].
An estimated 422 million people were affected by diabetes worldwide in 2014 [2]. The prevalence of diabetes has been steadily increasing for the past three decades, from an estimated 108 million in 1990 , and is growing most rapidly in low- and middle-income countries [3]. DR is the leading cause of vision loss of working-age adults, and DME is the most frequent cause of vision loss related to diabetes. The severity of visual impairment in DME varies depending on the type and duration of diabetes. The Wisconsin Epidemiologic Study of Diabetic Retinopathy found the 14-year incidence of DME 
in type 1 diabetics to be $26 \%$ [4]. Similarly, the Diabetes Control and Complications Trial reported that $27 \%$ of type 1 diabetic patients develop DME within nine years of onset [5]. An even higher incidence of macular edema has been reported in patients with type 2 diabetes [6].

Older onset patients have a tendency to develop macular edema earlier in the course of their disease (prevalence: 3-8\% with up to three years of disease duration) compared to younger onset patients (prevalence: $0.5 \%$ with up to 10 years of disease duration). In the presence of macular edema, $50 \%$ of older onset patients have visual acuity worse than 20/40 compared to $20 \%$ of younger onset patients [4].

Bevacizumab (Avastin) is a recombinant humanised monoclonal antibody that binds to the receptor binding sequence of diffusible vascular endothelial growth factor (VEGF) isoforms. This reduces macular edema and vascularity of epiretinal membranes. Classic clinical trials do not uniformly exist; however, convincing data has been published for the most commonly treated pathologies. Bevacizumab is considered as an efficacious for treatment by the ophthalmological community.

The aim of the study was to retrospectively evaluate the efficacy of intravitreal injections bevacizumab in a large group of DME patients.

\section{MATERIALS AND METHODS}

One hundred patients (125 eyes) were treated. The inclusion criteria consisted of the presence of diabetes mellitus type 1 or 2 , age over 35 years, and macular edema of $300 \mu \mathrm{m}$ or more. Presence of vitreoretinal traction, argon laser therapy in the past five months, or vitreoretinal surgery excluded patients from therapy. The average patient age was 60 years ( \pm 14 years). The group consisted of 48 women and 52 men. Only nine persons had type 1 diabetes; the others suffered from type 2 diabetes. Zeiss Stratus optical coherence tomography (OCT) was performed on qualification procedure (at least two weeks prior to the first injection) and three months after the first injection. The best corrected visual acuity was evaluated using the ETDRS charts on qualification procedure and during the examination in monthly intervals.

During the study two or three intravitreal drug injections were performed in 30-day intervals. OCT was performed on qualification procedure (at least two weeks prior to the first injection) and one month after the last injection. For three days be- fore and three days after injections, antibiotic drops were administered to the eye three times daily. The procedure was performed under local anaesthesia in the operating room after washing the conjunctiva with $1 \%$ povidone-iodine solution. The patients were treated with $50 \mu \mathrm{L}(1.25 \mathrm{mg})$ of bevacizumab administered to the vitreous body at a distance of $3.5-4 \mathrm{~mm}$ from the limbus.

\section{RESULTS}

The average thickness of macular retina was evaluated in OCT. It was marked as 457 microns ( \pm 183 microns) on the baseline examination. The mean (SD) best correct visual acuity on the initial examination was 63.8 ( \pm 26.2$)$ letters.

A month after the first injection a marked improvement in visual acuity in 57 eyes was observed; in 46 eyes visual acuity remained unchanged. Deterioration of visual acuity occurred in 22 eyes. One month after the second injection improvement occurred in 37 eyes, and in 63 visual acuity remained unchanged; deterioration occurred in 25 eyes. In patients who had not improved during the study, the third injection was discontinued. After the third injection in 42 eyes there was no improvement in visual acuity, of which in fifteen there was a deterioration. At Months 1, 2, and 3 the mean change (SE) in BCVA from baseline was $+4.4( \pm 0.49),+4.9( \pm 0.38)$, and $+3.1( \pm 0.39)$ letters, respectively.

The improvement of retinal morphology in OCT was spectacular: a decrease in the number of intraretinal cysts and a generalised shrinkage of retinal macular edema. The mean central macular thickness measured in OCT was 457 microns ( \pm 183 microns) on the qualification examination; after three months it dropped to 308 microns ( \pm 122 microns) (Fig. 1).

There were no serious adverse effects of the treatment. However, the following were observed:

- in $10.3 \%$ of patients - mild eye irritation;

- in $8.5 \%$ - ocular pain;

- in $1.7 \%$ - subconjunctival haemorrhage.

The average increase of intraocular pressure after injection was $3 \mathrm{~mm} \mathrm{Hg}( \pm 2.5 \mathrm{~mm} \mathrm{Hg})$.

\section{DISCUSSION}

Drugs currently registered for the treatment of DME are ranibizumab (Lucentis) and aflibercept (Eylea). Bevacizumab is a complete molecule of an anti-VEGF monoclonal antibody, and ranibizumab 

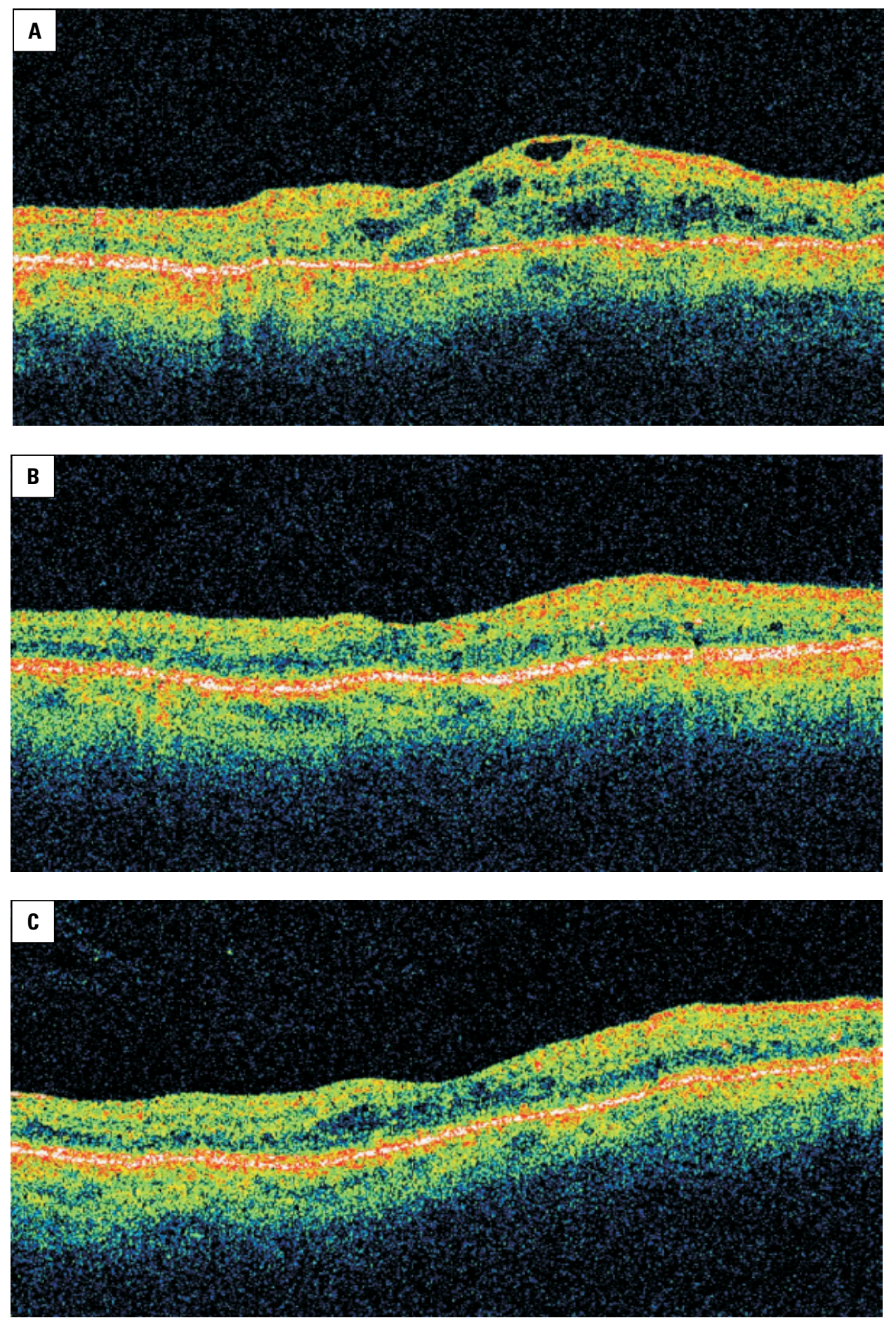

FIGURE 1. The OCT image of a 57-year-old patient suffering from diabetes type 2. Before treatment (A) diffuse retinal edema and the presence of fluid cysts can be seen. One month after the injection (B) and after three months (C) the image has been normalised. A significant reduction in number of fluid cysts and a generalised decrease of macular thickness can be observed. Source: the author's own materials (Department of Ophthalmology, Medical University of Gdansk, Poland)

is only an antigen binding fragment of the antibody. It means that bevacizumab persists longer in the vitreous chamber. It is emphasised that the duration of a single intravitreal injection effect is six weeks, but no treatment effects last longer than 12 weeks [7].
Recent studies have confirmed the greater improvement in visual acuity of patients treated with injections of anti-VEGF compared to laser photocoagulation. Although only $25 \%$ of patients took advantage of the therapy $[8,9]$. 
Comparing our outcome with the results of other studies is challenging. Most of the studies report the results after long-term treatment, i.e. 6, 12, 18, or 24 months [10-12]. Extracting data or drawing conclusions for a shorter period of observation is not always possible. The three-month outcome of this study was slightly worse than described in the Diabetic Retinopathy Clinical Research Network study [10]. One of the reasons might be that the baseline BCVA was higher in our study $63.8( \pm 26.2)$ vs. 56.6 ( \pm 10.6). Sivaprasad et al. [13] claim that patients with worse initial BCVA gained more letters at 12 months than those with better BCVA. Furthermore, in our study a drop of BCVA at week 12 was noted. The deterioration in such an early period, and despite ongoing treatment, differs from the results of other studies. Joshi et al. [14] claim that there might be a slight deterioration in visual acuity at 12 weeks of treatment, but only in eyes that show a decrease in central macula thickness $\geq 20 \%$. In our study such deterioration was observed on a large groups of patients regardless of the initial macular thickness. We believe that our results could call into question the sustainability of long-term anti-VEGF treatment.

There is high-quality evidence that antiangiogenic drugs provide benefit compared to other therapeutic options for DME, i.e. grid laser photocoagulation, or no treatment when laser is not an option [15]. The quality and quantity of the evidence was larger for ranibizumab, but there was little power to investigate drug differences [16]. Several socioeconomic studies emphasise the almost 30-fold difference in costs between bevacizumab and other FDA-approved anti-VEGF treatment $[17,18]$. This is to outline that during the study 292 intravitreal injections were administered, although at the time of the study the Polish National Health Fund did not reimburse the cost of any anti-VEGF agents in DME.

The use of anti-VEGF intravitreal treatment does not cause undesirable short-term side-effects. Similar results have been found in our study. The safety of anti-VEGF drugs is confirmed by studies conducted on large groups of patients suffering from age-related macular disease and DME [10-12, 19-21].

It may be questioned whether to expose patients to an off-label treatment since not all of them shall embrace the improvement of visual acuity. A treatment that leads to definite and permanent improvement in visual acuity in DME patients is yet unknown. Its progression is usually asymmetri$\mathrm{cal}$, and the occurrence of vision loss in one eye is likely to lead to a similar situation in the other eye. For patients whose visual acuity does not allow reading, we believe that it is worth taking the chance to improve the quality of vision.

\section{CONCLUSIONS}

Intravitreal injections of $1.25 \mathrm{mg}$ of bevacizumab each month in patients with DME caused a significant reduction of retinal edema.

In our study the majority of patients showed an improvement in visual acuity, particularly after the first injection. There were no serious adverse effects.

Bevacizumab therapy is an interesting alternative to DME treatment

\section{Conflict of interest: none declared}

\section{REFERENCES}

1. Ferris FL, Patz A. Macular edema. A complication of diabetic retinopathy. Surv Ophthalmol. 1984; 28 Suppl: 452-461, indexed in Pubmed: 6379946.

2. World Health Organization. Global Report On Diabetes 2016. http:// apps.who.int/iris/bitstream/10665/204871/1/9789241565257 eng.pdf?ua $=1$.

3. NCD Risk Factor Collaboration (NCD-RisC). Worldwide trends in diabetes since 1980: a pooled analysis of 751 population-based studies with 4.4 million participants. Lancet. 2016; 387(10027): 1513-1530, doi: 10.1016/S0140-6736(16)00618-8, indexed in Pubmed: 27061677.

4. Klein R, Klein B, Moss S, et al. The Wisconsin epidemiologic study of diabetic retinopathy: XVII. Ophthalmology. 1998; 105(10): 1801-1815, doi: 10.1016/s0161-6420(98)91020-x.

5. Progression of retinopathy with intensive versus conventional treatment in the Diabetes Control and Complications Trial. Diabetes Control and Complications Trial Research Group. Ophthalmology. 1995; 102(4): 647-661, indexed in Pubmed: 7724182.

6. Lee R, Wong TY, Sabanayagam C. Epidemiology of diabetic retinopathy, diabetic macular edema and related vision loss. Eye Vis (Lond). 2015; 2: 17, doi: 10.1186/s40662-015-0026-2, indexed in Pubmed: 26605370.

7. Yilmaz T, Cordero-Coma M, Gallagher MJ, et al. Systematic review of intravitreal bevacizumab injection for treatment of primary diabetic macular edema. Acta Ophthalmol. 2011; 89(8): 709-717, doi: 10.1111/j.1755-3768.2010.01918.x, indexed in Pubmed: 20645926.

8. Zechmeister-Koss I, Huic M. Vascular endothelial growth factor inhibitors (anti-VEGF) in the management of diabetic macular edema: a systematic review. Br J Ophthalmol. 2012; 96(2): 167-178, doi: 10.1136/ bjophthalmol-2011-300674, indexed in Pubmed: 22133986.

9. Michaelides M, Kaines A, Hamilton RD, et al. A prospective randomized trial of intravitreal bevacizumab or laser therapy in the management of diabetic macular edema (BOLT study) 12-month data: report 2. Ophthalmology. 2010; 117(6): 1078-1086.e2, doi: 10.1016/j.ophtha.2010.03.045, indexed in Pubmed: 20416952.

10. Wells JA, Glassman AR, Ayala AR, et al. Diabetic Retinopathy Clinical Research Network. Aflibercept, bevacizumab, or ranibizumab for diabetic macular edema. N Engl J Med. 2015; 372(13): 1193-1203, doi: 10.1056/NEJMoa1414264, indexed in Pubmed: 25692915.

11. Ahmadieh $H$, Ramezani $A$, Shoeibi $N$, et al. Intravitreal bevacizumab with or without triamcinolone for refractory diabetic macular edema; 
a placebo-controlled, randomized clinical trial. Graefes Arch Clin Exp Ophthalmol. 2008; 246(4): 483-489, doi: 10.1007/s00417-007-06880, indexed in Pubmed: 17917738.

12. Soheilian M, Ramezani A, Obudi A, et al. Randomized trial of intravitreal bevacizumab alone or combined with triamcinolone versus macular photocoagulation in diabetic macular edema. Ophthalmology. 2009; 116(6): 1142-1150, doi: 10.1016/j.ophtha.2009.01.011, indexed in Pubmed: 19376585.

13. Sivaprasad S, Crosby-Nwaobi R, Esposti SD, et al. Structural and functional measures of efficacy in response to bevacizumab monotherapy in diabetic macular edema: exploratory analyses of the BOLT study (report 4). PLoS One. 2013; 8(8): e72755, doi: 10.1371/journal. pone.0072755, indexed in Pubmed: 24013651.

14. Joshi L, Bar A, Tomkins-Netzer 0, et al. Intravitreal bevacizumab injections for diabetic macular edema - predictors of response: a retrospective study. Clin Ophthalmol. 2016; 10: 2093-2098, doi: 10.2147/ OPTH.S109809, indexed in Pubmed: 27799737.

15. Virgili G, Parravano M, Menchini $F$, et al. Anti-vascular endothelial growth factor for diabetic macular edema. Cochrane Database Syst Rev. 2014(10): CD007419, doi: 10.1002/14651858.CD007419.pub4, indexed in Pubmed: 25342124.

16. Virgili G, Parravano M, Menchini F, et al. Antiangiogenic therapy with anti-vascular endothelial growth factor modalities for dia- betic macular edema. Cochrane Database Syst Rev. 2012; 12: CD007419, doi: 10.1002/14651858.CD007419.pub3, indexed in Pubmed: 23235642.

17. A Systematic Review of Intravitreal Bevacizumab for the Treatment of Diabetic Macular Edema. https://www.ncbi.nlm.nih.gov/ pubmed/27195370.

18. Li E, Greenberg PB, Voruganti I, et al. Cost and Selection of Ophthalmic Anti-Vascular Endothelial Growth Factor Agents. R I Med J (2013). 2016; 99(5): 15-17, indexed in Pubmed: 27128510.

19. Ladas ID, Karagiannis DA, Rouvas AA, et al. Safety of repeat intravitreal injections of bevacizumab versus ranibizumab: our experience after 2,000 injections. Retina. 2009; 29(3): 313-318, doi: 10.1097/ IAE.0b013e31819a5f98, indexed in Pubmed: 19287287.

20. Fintak DR, Shah GK, Blinder KJ, et al. Incidence of endophthalmitis related to intravitreal injection of bevacizumab and ranibizumab. Retina. 2008; 28(10): 1395-1399, doi: 10.1097/IAE.0b013e3181884fd2, indexed in Pubmed: 18827737.

21. Wu L, Martínez-Castellanos MA, Quiroz-Mercado H, et al. Pan American Collaborative Retina Group (PACORES). Twelve-month safety of intravitreal injections of bevacizumab (Avastin): results of the Pan-American Collaborative Retina Study Group (PACORES). Graefes Arch Clin Exp Ophthalmol. 2008; 246(1): 81-87, doi: 10.1007/s00417007-0660-z, indexed in Pubmed: 17674014. 